\title{
Performance of Asymmetric Slot -Entry Hybrid Journal Bearings
}

\author{
A. Abdelbadie, W.A. Crosby, I.M. ElFahham
}

\begin{abstract}
A comparative study of slot - entry hybrid journal bearings performance with different symmetric and asymmetric geometrical configurations has been presented. The research work includes a numerical analysis of full thermo-hydrodynamic non-recessed slot entry journal bearing. The results show the optimum number of slots for each row of double row symmetric configuration and for single row asymmetric case. The results demonstrated that the optimized asymmetric configuration has higher load carrying capacity especially at high eccentricity ratio. The effect of temperature rise on load carrying capacity of the bearing is more pronounced in symmetric than that in asymmetric configuration. Considering the stability of the bearing, the comparative study indicates that asymmetric geometric configurations have better stability compared to symmetric geometric configurations with higher threshold speed margin.
\end{abstract}

Keywords - Hybrid Bearing, slot-entry, asymmetric bearing, stability

\section{Introduction}

Hybrid journal bearing operates under combined hydrodynamic action and pressurized lubricant. By combining hydrostatic and hydrodynamic built pressure, they are capable of supporting high loads and speeds. Hybrid journal bearings are mainly categorized according to the lubricant entry into hole-entry and slot-entry as illustrated in Figure 1. The entry end is also categorized into recessed or non-recessed end as shown in Figure 2. Generally, in plain hybrid bearings it is recommended to avoid recesses in order to generate more hydrodynamic effect at high speeds. The bearing configurations can also be categorized into symmetric or asymmetric as will be shown in Mathematical Modeling section. The geometry of the bearing lubricant entry has been the subject of many research works [1-5]. Satish C.Sharma [2, 3, 5] studied the performance of hydrostatic and hybrid journal bearing compensated by slot restrictor. The theoretical results indicated that using asymmetric slot-entry journal bearings a- Slot-Entry

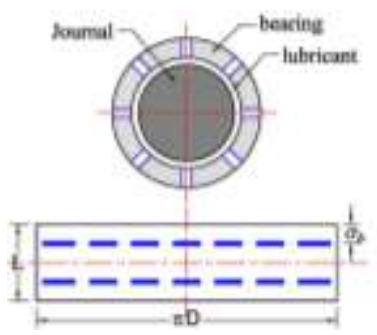

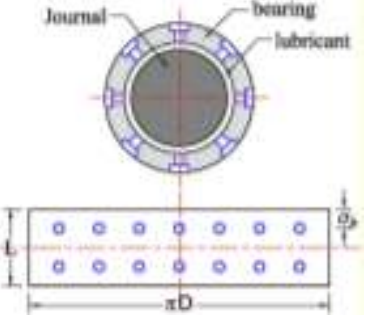

b- Hole-Entry
Fig. 1 Hybrid journal bearing configurations

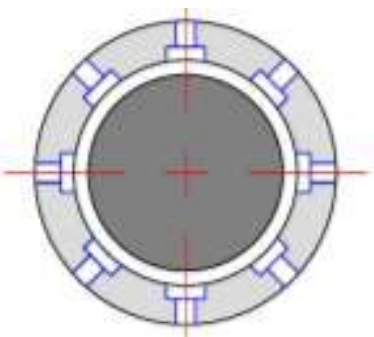

a- Recessed bearing

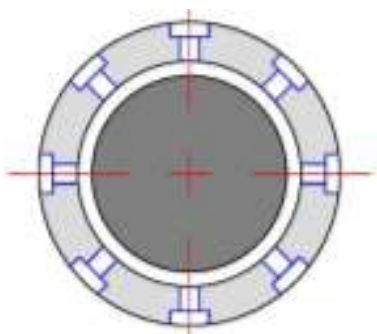

b- Non-recessed bearing

Fig. 2 Hybrid journal bearing entry end configurations

can improve the stability of journal bearings when proper selection of the bearing parameter and type of bearing configuration is considered. Zhang [6] conducted a numerical analysis to investigate the power consumption in different types of fluid film bearings. He found that the hybrid configuration had the lowest consumption. Hsien and Huang [7] studied hybrid bearings with single-row, 6 slots with orifice compensation. Results indicate that, restriction parameter and land-width ratio are important factors that affect the load carrying capacity and the stability of the systems. Mancilla and Navarro [8] also studied the imbalance response of a rotor supported by a hybrid bearing. The results show an increase in velocity thresholds and a decrease in vibration amplitude compared to the conventional circular bearings. Garg [9] presented a theoretical study of the lubricant rheological effects on the performance of hybrid journal bearing. He used an asymmetric slot-entry configuration and found out that the viscosity variation due to temperature rise decrease the bearing performance quite significantly. Vikas and Satish [10] presented a comparative study for hybrid journal bearing with 2-lobe multi-recess. The results indicated that suitable type of flow control compensating devices should be selected to improve the bearing performance.

The present research work considers the load carrying capacity and stability of hybrid journal bearings. This theoretical analysis studies the thermal effects and stability threshold for slot-entry hybrid journal bearing with symmetric and asymmetric configurations.

\section{NOMENCLATURE}

\section{DIMENSIONAL PARAMETERS}

\author{
$A$ : Area $\left(m^{2}\right)$ \\ $a b:$ Axial land width $(\mathrm{m})$ \\ as : Extent of slot (m) \\ $\zeta$ : Viscosity index coefficient
}


$c$ : Radial clearance (m)

$C_{p}:$ Specific heat of fluid $\left(J . \mathrm{kg}^{-1} \cdot K^{-1}\right)$

$C_{i j}$ : Fluid-film damping coefficients $\left(N . m^{-2}\right)$

$D:$ Journal diameter $(\mathrm{m})$

$e:$ Journal eccentricity (m)

$f$ : Fluid film

$J$ : Journal

$h$ : Fluid-film thickness (m)

$\mathrm{h}_{\min }$ : Minimum fluid-film thickness (m)

$h_{\mathrm{b}}$ : Heat transfer coefficient for the bush $\left(W \cdot m^{-1} \cdot K^{-1}\right)$

$k_{\mathrm{b}}$ : Thermal conductivity for the bush $\left(W \cdot m^{-1} \cdot K^{-1}\right)$

$k_{\mathrm{f}}$ : Thermal conductivity for the fluid $\left(W \cdot m^{-1} \cdot K^{-1}\right)$

$L$ : Length of bearing (m)

$M$ : Mass $(\mathrm{kg})$

$N$ : Rotational speed ( rpm)

$p:$ Pressure $\left(N . m^{-2}\right)$

$p_{s}:$ Supply Pressure $\left(N . m^{-2}\right)$

$p^{*}$ : Pressure at concentric condition i.e at $\varepsilon=0.0$.

${ }_{R}:$ Restrictor

$R_{1}, R_{2}$ : Inner and outer bush radius (m)

$r:$ Radial coordinate

$r_{J}$ : Journal radius $(\mathrm{m})$

Sij: Fluid-film stiffness coefficients $(i, j=\mathrm{x}, \mathrm{y})\left(N . m^{-1}\right)$

$t$ : Time (sec.)

$T$ :Temperature $\stackrel{0}{C}$

$T_{a}$ : Ambient temperature $\stackrel{0}{C}$

$T_{f}$ : Film temperature $\stackrel{0}{C}$

$T_{J}$. Journal temperature $\stackrel{0}{C}$

$T_{b}$ : Bush temperature $\stackrel{0}{C}$

$u, v, w$ : Fluid velocity components in $\mathrm{X}, \mathrm{Y}, \mathrm{Z}$ direction $\left(m \cdot \sec ^{-1}\right)$

$x$ : Circumferential Cartesian coordinate

$y:$ Coordinate along film thickness

z: Axial Cartesian coordinate
$W_{x}$ : Load carrying capacity in $\mathrm{x}$ direction

$W_{y}$ : Load carrying capacity in y direction

$W_{o}$ : External load $(\mathrm{N})$

$X, Y, Z:$ Cartesian coordinate system

$X_{J}, Z_{J}$ : Journal center coordinate

$Y s$ : Radial length of slot (m)

$Z s:$ Axial width of slot (m)

$\rho$ : Density $\left(\mathrm{kg} \cdot \mathrm{m}^{-3}\right)$

$\mu$ : Lubricant viscosity ( Pa.s)

$\mu_{a}$ : Apparent viscosity (Pa.s)

$\mu_{r}:$ Reference viscosity (Pa .s)

$\omega$ : Journal rotational speed $\left(\mathrm{sec}^{-1}\right)$

$\theta$ : Circumferential cylindrical coordinate

$\beta$ : Axial cylindrical coordinate

$\phi$ : Attitude angle (deg.)

$\alpha$ : Coefficient of thermal conductivity

$\psi$ : Coefficient of discharge for orifice

\section{DIMENSIONALESS PARAMETERS}

$\bar{a}_{b}:$ Land width ratio $=a_{b} / L$

$\bar{q}_{j \backslash i n}:$ Heat flow to the journal

$\bar{q}_{j \backslash o}:$ Heat flow from the journal

$\bar{\beta}^{*}:$ Concentric design pressure ratio $=p^{*} / p_{s}$

$\bar{p}$ :Dimensionless pressure $=p / p_{s}$

$\lambda:$ ratio of $(\mathrm{L} / \mathrm{D})$

$S W R=$ Slot width ratio $=\left(a_{s} / a_{s \max }\right)=\left(a_{s} n / \pi D\right)$

$\overline{C_{s R}}=$ Restrictor design parameter

$=\frac{\pi}{36} \frac{S W R}{\lambda} \frac{k}{a_{b}}\left(\frac{a_{b}}{Y_{s}}\right)\left(\frac{Z_{s}}{c}\right)^{3} \ldots \ldots \ldots . .$. for slot

$$
=\frac{1}{128}\left(\frac{\pi d^{4}{ }_{c a p}}{c^{3} L_{c a p}}\right)
$$
for capillary 
Proc. of Seventh International Conference On Advances in Civil, Structural and Mechanical Engineering -ACSM 2017 Copyright (C) Institute of Research Engineers and Doctors, USA .All rights reserved.

ISBN: 978-1-63248-135-1 doi: 10.15224/ 978-1-63248-135-1-45

$=\frac{1}{12}\left(\frac{3 \pi d_{o r i}^{2} \mu_{r} \psi}{c^{3}}\right)\left(\frac{2}{\rho p_{s}}\right)^{0.5} \ldots \ldots$ for orifice

$k$ :Number of rows of slots/holes in a bearing.

$n:$ Number of nodes, holes/slots,

$\varepsilon:$ Eccentricity ratio $=e / c$

$\bar{T}:$ Dimensionless temperature $=\left(T-T_{0}\right) / T_{0}$

$y$ : Dimensionless Coordinate along film thickness $=y / h$

$p_{e}:$ Peclet number $=\left(\rho C_{p} \omega c^{2}\right) / \alpha$

$\bar{u}$ : Dimensionless velocity of the journal $=u /(\omega r)$

$\bar{h}$ : Dimensionless fluid-film thickness $=h / c$

$\bar{\mu}$ : Dimensionless viscosity $=\mu / \mu_{0}$

$\overline{k_{b}}$ : Dimensionless thermal conductivity for the

bush $=k_{b} / k_{r}$

$\bar{T}_{b}:$ Dimensionless bush temperature $=T_{b} / T_{0}$

$r$ : Dimensionless radial coordinate $=r / r_{j}$

$\beta=z / r_{j}, \theta=x / r, \bar{z}=z / L$,

$\Omega=\omega\left(\mu_{r} r^{2}\right) /\left(c^{2} p_{s}\right)$

\section{Mathematical Model}

The mathematical model for non-recessed slot entry hybrid journal bearings starts with the generalized Reynolds equation for fluid flow with variable viscosity. Once the pressure distribution is evaluated, the energy equation is used to determine the temperature distribution within the fluid film. Then, the 3D heat conduction equation is used to compute the temperature rise in the bush.

\subsection{Bearing geometry:-}

The bearings considered in this study have circular shape with non-recessed slot entry. For comparative analysis, different geometries are used such as symmetry and asymmetry configurations with single and double rows as shown in Figure 3. The number of slots entry ranged from 4 to 24 slots per row. Different operating parameters are considered such as length to diameter ratio, eccentricity ratio, land width ratio and restrictor design parameter.
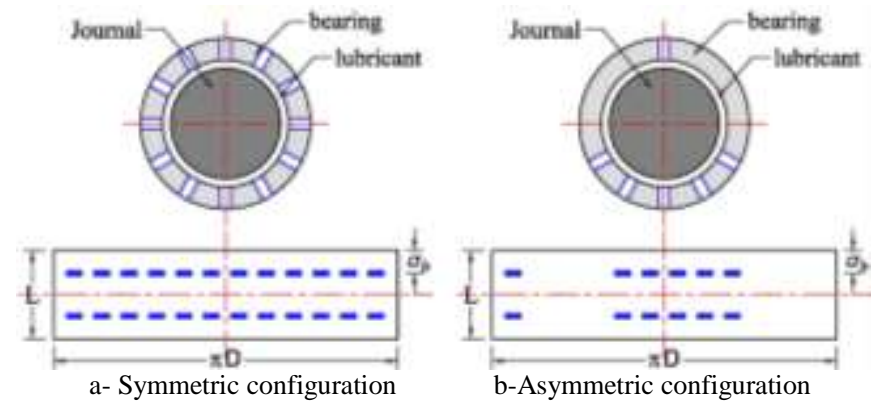

Fig. 3 Slot-Entry hybrid journal bearing configurations

\subsection{Generalized Reynolds Equation:-}

Considering that the bearing geometry in Cartesian coordinates $\mathrm{X}, \mathrm{Y}, \mathrm{Z}$ were taken in circumferential direction, across the film thickness and along the axis of the bearing respectively. The circumferential direction is then considered in circumferential cylindrical coordinate to be equal to $\theta \mathrm{R}$. The journal rotates with angular velocity $\omega$ as shown in Figure 4. The generalized Reynolds equation for laminar flow of incompressible fluids was used as governing equation for the lubricant flow in the clearance space of journal and bearing as presented by Dowson [1] in the following form:

$\frac{\partial}{\partial \theta}\left(\bar{h} \bar{k}_{2} \frac{\partial \bar{p}}{\partial \theta}\right)+\frac{\partial}{\partial \bar{z}}\left(\bar{h} \bar{k}_{2} \frac{\partial \bar{p}}{\partial \bar{z}}\right)=\Omega \frac{\partial}{\partial \theta}\left(\left(1-\frac{\overline{k_{1}}}{\bar{k}_{0}}\right) \bar{h}\right)+\frac{\partial \bar{h}}{\partial \bar{t}}$

Where the dimensionless viscosity function $\bar{k}_{0}, \bar{k}_{1}, \bar{k}_{2}$ can be expressed as:

$\bar{k}_{0}=\int_{0}^{1} \frac{1}{\bar{\mu}} d \bar{y}, \quad \bar{k}_{1}=\int_{0}^{1} \frac{\bar{y}}{\bar{\mu}} d \bar{y} \quad, \quad \overline{k_{2}}=\int_{0}^{1} \frac{\bar{y}}{\bar{\mu}}\left(\bar{y}-\frac{\overline{k_{1}}}{\overline{k_{0}}}\right) d \bar{y}$

The boundary conditions are considered as follows:-

a. Nodes on the bearing boundary have zero pressure.

$$
p_{( \pm L / 2)}=0.0
$$

b. The restrictor flow $\bar{q}_{r}$ is equal to input flow of bearing at slot.

c. Both pressure and pressure gradient in circumferential direction at the trailing edges equal zero.

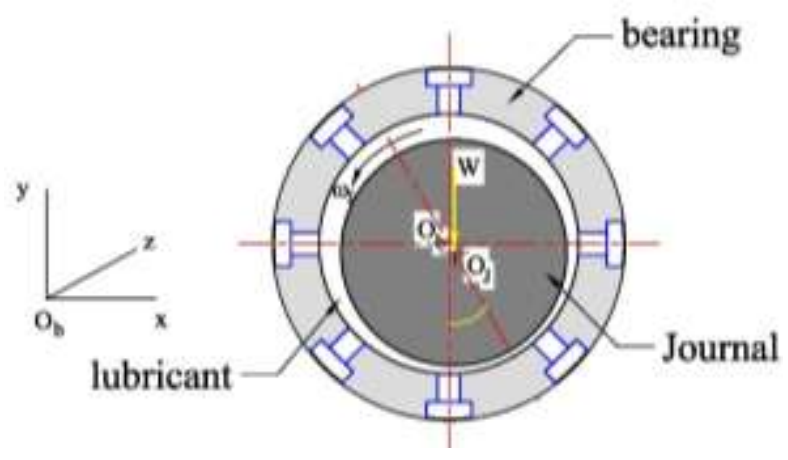

Fig. 4 Geometric details and coordinate system 


$$
p=0.0, \frac{\partial p}{\partial \theta}=0.0
$$

The pressure dependent viscosity is defined in dimensionless form as [11].

$\bar{\mu}=\frac{\mu}{\mu_{0}}$ Where $\bar{\mu}=\exp \left(-\zeta\left(T-T_{0}\right)\right)$

The fluid film thickness for a journal bearing in nondimensional form is expressed as follows:

$$
\bar{h}=1-\bar{X}_{j} \cos \alpha-\bar{Z}_{j} \sin \alpha
$$

\subsection{Flow Equation for Restrictor:-}

Flow control devices are usually used with externally pressurized non-recessed journal bearing. The lubricant flow in most common flow control devices is expressed in nondimensional forms as $[2,12]$

$$
\begin{array}{ll}
\text { 1.Capillary } & \bar{q}_{r}=\bar{C}_{s R}\left(1-\bar{p}_{r}\right) \\
\text { 2.Orifice } & \bar{q}_{r}=\overline{C_{s R}}\left(1-\bar{p}_{r}\right)^{0.5}
\end{array}
$$

3. Constant flow valve $\overline{q_{r}}=\overline{q_{c}}$

4.Slot entry restrictors $\bar{q}_{r}=\bar{C}_{s R}\left(1-\bar{p}_{r}\right)$

Where: $\bar{p}_{r}$ is the dimensionless pressure at bearing slot.

$\bar{C}_{s R}$ is the slot-restrictor design parameter and is given by:

$$
\overline{C_{s R}}=\frac{\pi}{36} \frac{S W R}{\lambda} \frac{k}{\bar{a}_{b}}\left(\frac{a_{b}}{Y_{s}}\right)\left(\frac{Z_{s}}{c}\right)^{3}
$$

Where: $\quad \bar{a}_{b}=a_{b} / \mathrm{L}=$ land width ratio,

$K=$ number of rows of slots in a bearing.

(SWR) is called slot width ratio and is defined as the ratio between the actual slot width " $a_{s}$ " and the maximum available slot width $\left(a_{s}\right)_{\max }$ as shown below:

$$
S W R=\frac{a_{s}}{\left(a_{s}\right)_{\max }}=\frac{a_{s} n}{\pi D}
$$

In the case of $\varepsilon=0.0$ which is the concentric condition:

$\bar{\beta}^{*}=p^{*} / p_{s}=$ concentric design pressure ratio (the dimensionless pressure ratio at concentric condition).

$$
\overline{C_{s R}}=\frac{1}{k n} \frac{\pi}{6 \lambda \overline{a_{b}}}\left(\frac{\bar{\beta}^{*}}{1-\bar{\beta}^{*}}\right)
$$

\subsection{Energy Equation:-}

The full 3-D energy equation in fluid film lubrication is the result of energy balance between the heat generated and the heat transferred. To determine the temperature distribution for incompressible lubricant, the energy equation is given as follows [9]:

$\rho C_{p}\left(u \frac{\partial T_{f}}{\partial x}+v \frac{\partial T_{f}}{\partial y}+w \frac{\partial T_{f}}{\partial z}\right)=\frac{\partial}{\partial x}\left(\alpha \frac{\partial T_{f}}{\partial x}\right)+\frac{\partial}{\partial y}\left(\alpha \frac{\partial T_{f}}{\partial y}\right)+\frac{\partial}{\partial z}\left(\alpha \frac{\partial T_{f}}{\partial z}\right)+\mu \psi$

Where $\psi$ is the dissipation function and is given as follows:-

$\psi=2\left(\frac{\partial u}{\partial x}\right)^{2}+2\left(\frac{\partial v}{\partial y}\right)^{2}+2\left(\frac{\partial w}{\partial z}\right)^{2}+\left(\frac{\partial u}{\partial y}+\frac{\partial v}{\partial x}\right)^{2}+\left(\frac{\partial v}{\partial z}+\frac{\partial w}{\partial y}\right)^{2}+\left(\frac{\partial w}{\partial x}+\frac{\partial u}{\partial z}\right)^{2}$

The fluid temperature variation in the axial direction is very small and can be neglected

$$
\text { So } \quad \frac{\partial T_{f}}{\partial z}=0.0
$$

Then, the above energy equations (13) reduced to the following non- dimensional energy equation:

$$
\frac{1}{p_{e}} \frac{\partial^{2} \bar{T}_{f}}{\partial \bar{y}^{2}}+\bar{h}^{2}\left(\bar{u} \frac{\partial \bar{T}_{f}}{\partial \theta}+\frac{\bar{v}}{\bar{h}} \frac{\partial \bar{T}_{f}}{\partial \bar{y}}\right)-\bar{u} \bar{y} \bar{h} \frac{\partial \bar{h}}{\partial \theta} \frac{\partial \bar{T}_{f}}{\partial \bar{y}}+\bar{\mu}\left(\left(\frac{\partial \bar{u}}{\partial \bar{y}}\right)^{2}+\left(\frac{\partial \bar{w}}{\partial \bar{y}}\right)^{2}\right)=0.0
$$

The boundary conditions used to solve the energy equation (16) are as follows:-

1.on the fluid-film journal interface

$$
\bar{T}_{f}=\bar{T}_{j} \quad \text { at } \quad \bar{y}=1.0
$$

2.on the fluid-film bush interface

$$
\bar{T}_{f}=\bar{T}_{b} \quad \text { at } \quad \bar{y}=0.0
$$

\subsubsection{Heat conduction:-}

The temperature distribution in the bush is determined by 3D Fourier heat conduction equation. This heat conduction equation in cylindrical coordinates with non-dimensional form is given as:

$$
\frac{1}{\bar{r}} \frac{\partial}{\partial \bar{r}}\left(\bar{k}_{b} \bar{r} \frac{\partial \bar{T}_{b}}{\partial \bar{r}}\right)+\frac{1}{\bar{r}^{2}} \frac{\partial}{\partial \theta}\left(\bar{k}_{b} \frac{\partial \bar{T}_{b}}{\partial \theta}\right)+\bar{r} \frac{\partial}{\partial \beta}\left(\bar{k}_{b} \frac{\partial \bar{T}_{b}}{\partial \beta}\right)=0.0
$$

The thermal boundary conditions for the solution of equation (19) are expressed as follows:

a. at the fluid-bush interface $\bar{y}=0.0, \quad \bar{r}=\bar{R}_{1}$ 


$$
-k_{b}\left(\frac{\partial \overline{T_{b}}}{\partial \bar{r}}\right)_{r=R_{1}}=\frac{k_{f}}{\bar{c} \bar{h}}\left(\frac{\partial \overline{T_{f}}}{\partial \bar{y}}\right)_{\bar{y}=0.0}
$$

b. On the outer faces of the bush $\quad \bar{r}=\bar{R}_{2}$ the free convection gives:

$$
-\left(\frac{\partial \bar{T}_{b}}{\partial \bar{r}}\right)_{\overline{r=R 2}}=-\frac{h_{b} R}{k_{b}}\left(\left.\bar{T}_{b}\right|_{r=R 2}-\bar{T}_{a}\right)
$$

c. On the lateral faces of the bearing $\beta= \pm L / 2$

$$
\left(\frac{\partial \bar{T}_{b}}{\partial \beta}\right)_{\beta= \pm L / 2}=-\frac{h_{b} R}{k_{b}}\left(\left.\bar{T}_{b}\right|_{\beta= \pm L / 2}-\bar{T}_{a}\right)
$$

d. At the supply slots of inlet edge, the temperature $\left(\bar{T}_{b}\right)$

is taken equal to the oil supply temperature $\left(\bar{T}_{s}\right)$.

\subsubsection{Journal temperature:-}

The temperature is considered uniform in the circumferential direction due to the continuous rotation of journal. In the case of thermal equilibrium, the heat flows to the journal equal to the heat flows from the journal. The amount of heat received by the journal is given by:

$\bar{q}_{\left.j\right|_{\text {in }}}=\left.\int_{L / 1}^{+L / 2} \int_{0}^{2 \pi} \frac{k_{f} R_{j}}{\bar{c} \bar{h}}\right|_{y=1} d \theta d \beta$

And the heat rejected through the two ends of the journal is given by

$\bar{q}_{j \mid o}=\frac{2 h_{j} A_{j}}{R_{j}}\left(\bar{T}_{j}-\bar{T}_{a}\right)$

Then the journal temperature $\left(\bar{T}_{j}\right)$ can be obtained by equating the equations (23) and (24).

\subsection{Stability parameters:-}

To start stability analysis, a very small disturbance is assumed in the center of the journal from its equilibrium position. The equation of motion for center of the journal can be given in the non-dimensional form as the follows:

$$
\left[\bar{M}_{J}\right]\left\{\tilde{X}_{J}\right\}+[\bar{C}]\left\{\dot{X}_{J}\right\}+[K]\left\{\bar{X}_{J}\right\}=0.0
$$

By solving equation (25), the stability margin of the system

for the critical mass $\left(\bar{M}_{c}\right)$ can be obtained.

If $\bar{M}_{J}<\bar{M}_{c}$ then, the system is stable.

Then, the dimensionless threshold speed of the journal can be obtained from the following relation:

$$
\begin{aligned}
& \bar{\omega}_{t h}=\left[\bar{M}_{c} / \bar{W}_{0}\right]^{0.5} \\
& \text { Where, }\left(\bar{W}_{0}\right) \text { is the reaction force at }\left(\frac{\partial \bar{h}}{\partial \bar{t}}=0.0\right)
\end{aligned}
$$

\section{Results and Discussions:-}

The hybrid bearing performance relies on many parameters such as length to diameter ratio, eccentricity ratio and land width ratio. The representative values of such parameters have been computed according to lubricant's properties, operating and geometric parameters of the bearing as shown in Table 1. The variation in dimensionless load carrying capacity vs. eccentricity ratio in the cases of full thermohydrodynamic and isothermal analysis has been presented in Figure 5 for both symmetric and asymmetric configurations. It is clear that the load carrying capacity is reduced in the thermo hydrodynamic solutions. This is due to the temperature rise, where the viscosity decreases, resulting in a decrease of the minimum film thickness leading to a reduction in the load carrying capacity. The change in loadcarrying capacity in full thermo-hydrodynamic solution, due to temperature variation, is more pronounced at high eccentricity ratio. It is also observed that those variations are practically similar in both geometrical configurations.

Figure 6 and 7 shows the variation of dimensionless load carrying capacity versus eccentricity ratio for single row with 6 and 8 slots. Figures 8 to 10 also show this relation for symmetric and asymmetric configuration with double rows in the cases of $12,8,6$ slots/row. The results clarifies that the asymmetric configurations give higher load carrying capacity than in the symmetric configurations for all cases.

Figure 11shows the Figure 11 variation of dimensionless load carrying capacity versus eccentricity ratio for bearing with symmetric configuration in single and double rows. It is found that bearing with double rows and 6 slots /row gives higher load carrying capacity than that in the single row with 12 slots.

The relation between the number of slot per row and the load carrying capacity at eccentricity ratio 0.7 are further shown in Figure 12 and 13. It is clear that the optimum number of slots per row for the case of symmetry with double rows is 12 slots. It is also found that the optimum number of slots per row for the case of asymmetry with single row is 6 slots. Figure 14 shows the relation between slot-restrictor design parameter and the dimensionless maximum pressure in both isothermal and full thermohydrodynamic solutions. This Figure illustrates the relation for the case of double rows with 6 slots in each row with symmetric and asymmetric configurations. The maximum dimensionless pressure is reduced in the full thermohydrodynamic solutions. Figure 15 also shows the relation between slot-restrictor design parameter and the dimensionless load carrying capacity in both isothermal and full thermo hydrodynamic solutions. The results demonstrated that the performance of the hybrid bearings depends on the use of optimum control parameters whereas 
the asymmetric configuration is generally advantageous compared to the symmetric configuration. Figure 16 illustrates the changes of threshold speed $\left(\bar{\omega}_{t h}\right)$ due to the variation of the speed parameter ( $\Omega$ ) for both the symmetric and asymmetric configurations. The stability margin for this threshold speed is shown in the Figure. The region below the curves is stable while the region above the curves is unstable. It is clear that, the bearing with asymmetric configuration is more stable than that of symmetric configuration. The computed results of the present work agree with the previous other studies belonged to hybrid journal bearing.

TABLE 1

BEARING DATA

\begin{tabular}{|c|c|c|c|}
\hline parameter & $\begin{array}{c}\text { symb } \\
\text { ol } \\
\end{array}$ & Value & $\overline{~ U n i t s ~}$ \\
\hline Bearing length & $L$ & 100 & $\mathrm{~mm}$ \\
\hline journal radius & $r$ & 50 & $m m$ \\
\hline Radial clearance & $c$ & 0.05 & $m m$ \\
\hline Land width ratio & $a_{b} / L$ & 0.25 & \\
\hline Density & $\rho$ & 850 & kg. $\mathrm{m}^{-3}$ \\
\hline $\begin{array}{l}\text { Specific heat of } \\
\text { fluid }\end{array}$ & $C_{p}$ & 2000 & $\begin{array}{l}J . \mathrm{kg}^{-} \\
{ }^{l} . K^{-1} \\
\end{array}$ \\
\hline $\begin{array}{l}\text { Thermal } \\
\text { conductivity of } \\
\text { fluid }\end{array}$ & $k_{f}$ & 0.125 & $\begin{array}{l}W \cdot m^{-} \\
{ }^{l} \cdot K^{-1}\end{array}$ \\
\hline $\begin{array}{l}\text { Thermal } \\
\text { conductivity of } \\
\text { bush }\end{array}$ & $k_{\mathrm{b}}$ & $50 W \cdot m^{-1} \cdot K^{-1}$ & $\begin{array}{l}W \cdot m^{-} \\
{ }^{l} . K^{-1}\end{array}$ \\
\hline Viscosity & $\mu$ & 0.0277 & Pa.s \\
\hline $\begin{array}{l}\text { viscosity index } \\
\text { coefficient }\end{array}$ & $\zeta$ & 0.01 & \\
\hline Journal speed & $N$ & 2500 & rpm \\
\hline External load & $\overline{W_{o}}$ & 22.4 & $k N$ \\
\hline Supply pressure & $p_{s}$ & $9 * 10^{6}$ & $N . m^{-2}$ \\
\hline Supply temperature & $T$ & $40^{\circ} \mathrm{C}$ & ${ }^{\circ} \mathrm{C}$ \\
\hline $\begin{array}{l}\text { Ambient } \\
\text { temperature }\end{array}$ & $T_{\mathrm{a}}$ & $40^{\circ} \mathrm{C}$ & ${ }^{\circ} \mathrm{C}$ \\
\hline
\end{tabular}

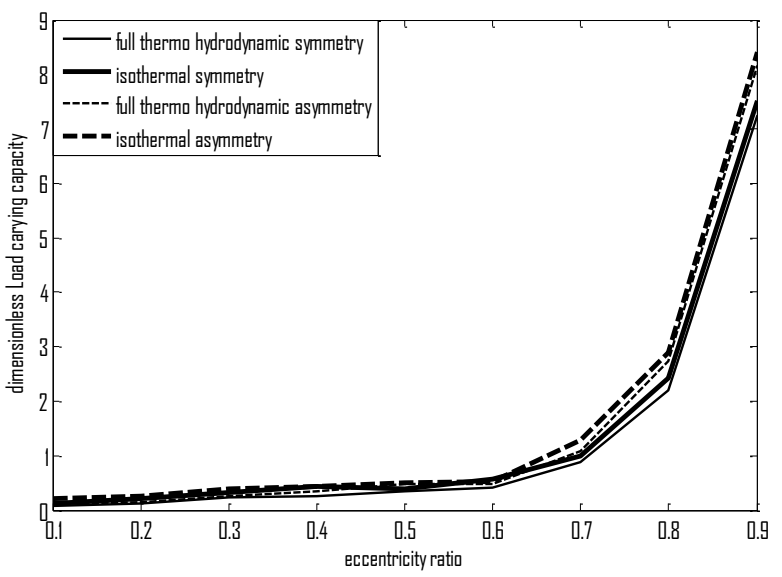

Fig. 5: Variation of dimensionless load carrying capacity vs eccentricity ratio with different configurations for full thermo-hydrodynamic and isothermal solutions

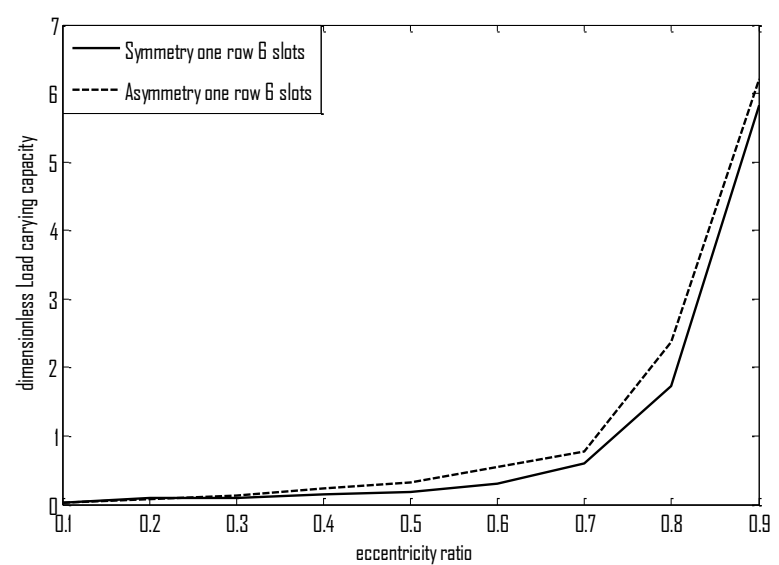

Fig. 6: Variation of dimensionless load carrying capacity vs eccentricity ratio for symmetric - asymmetric configuration with single row 6 slots

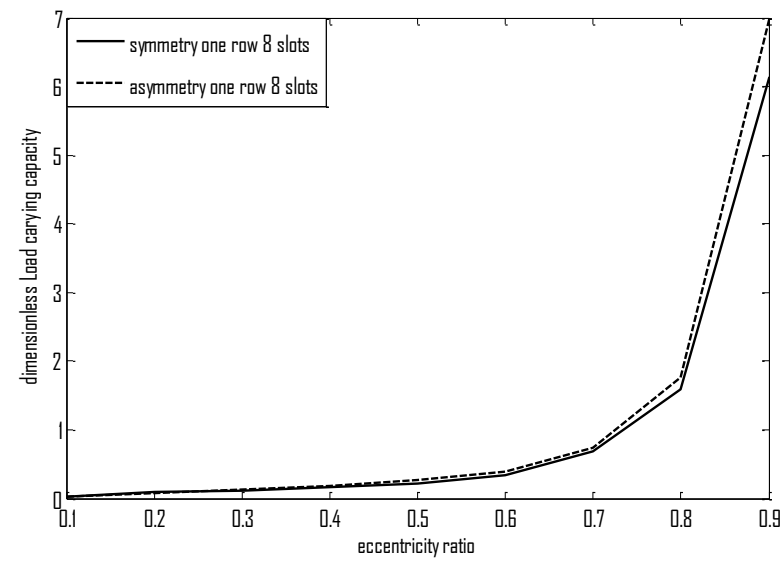

Fig. 7: Variation of dimensionless load carrying capacity vs eccentricity ratio for symmetric - asymmetric configuration with single row 8 slots

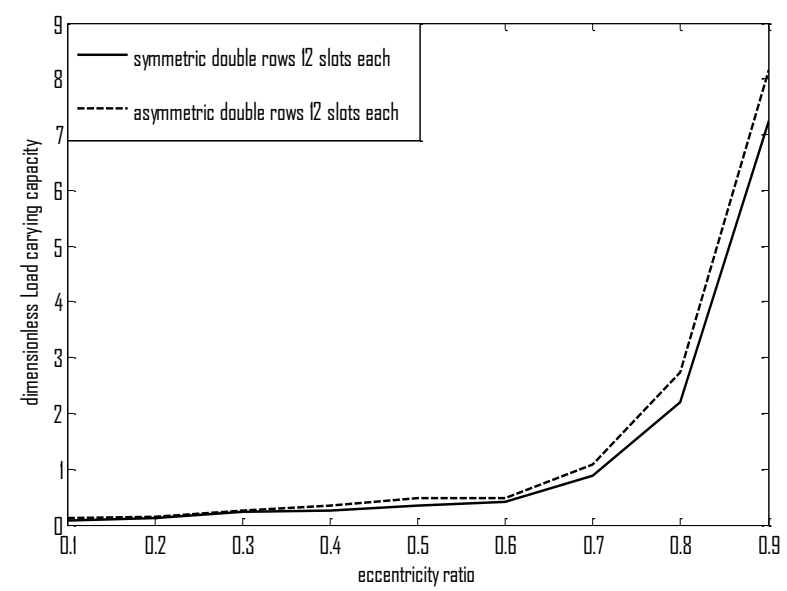

Fig. 8: Variation of dimensionless load carrying capacity vs eccentricity ratio for symmetric - asymmetric configuration with double row 12 slots 
Proc. of Seventh International Conference On Advances in Civil, Structural and Mechanical Engineering -ACSM 2017 Copyright (C) Institute of Research Engineers and Doctors, USA .All rights reserved.

ISBN: 978-1-63248-135-1 doi: 10.15224/ 978-1-63248-135-1-45

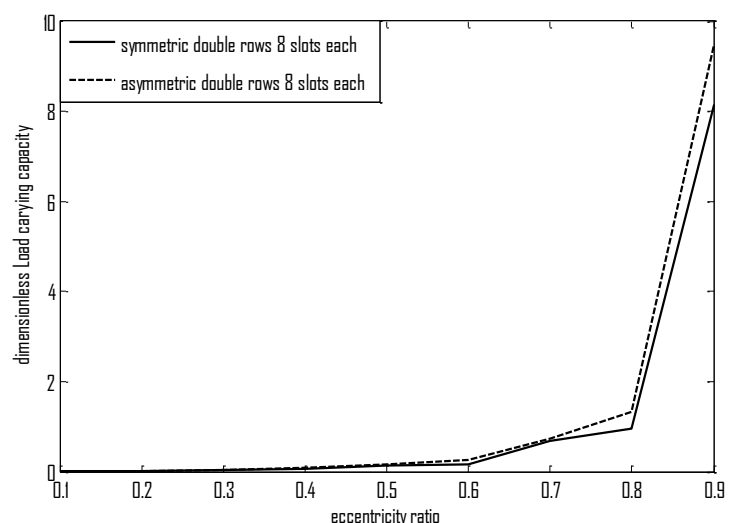

Fig. 9: Variation of dimensionless load carrying capacity vs eccentricity ratio for symmetric - asymmetric configuration with double row 8 slots

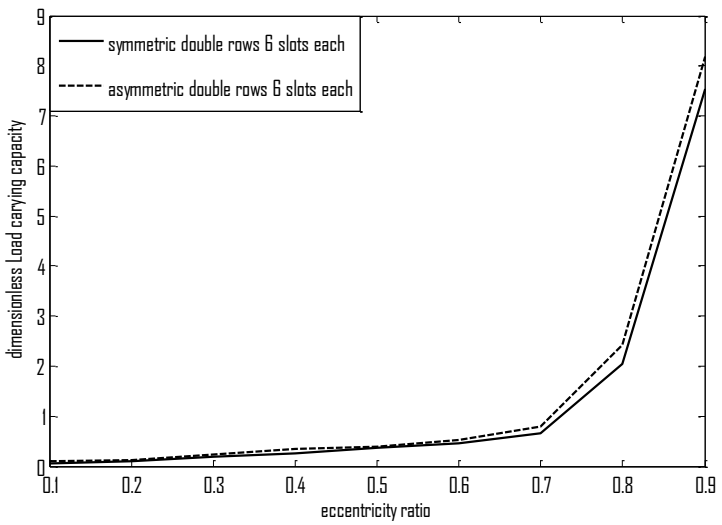

Fig. 10: Variation of dimensionless load carrying capacity vs eccentricity ratio for symmetric - asymmetric configuration with double row 6 slots

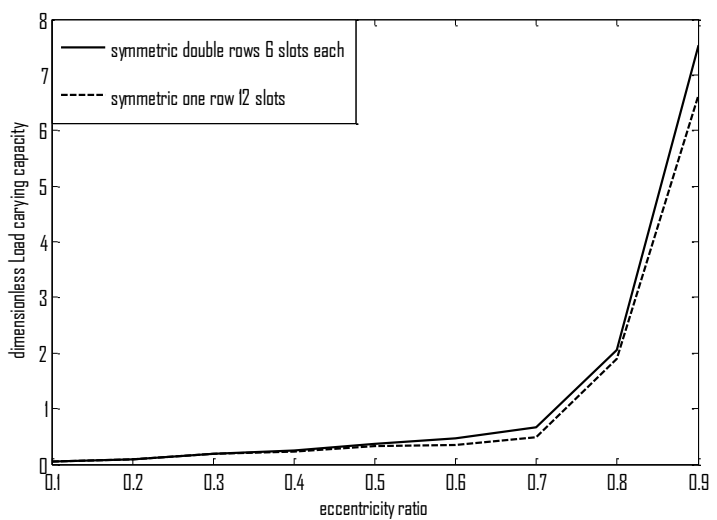

Fig. 11: Variation of dimensionless load carrying capacity vs eccentricity ratio for symmetric configuration with single and double rows 12,6 slots

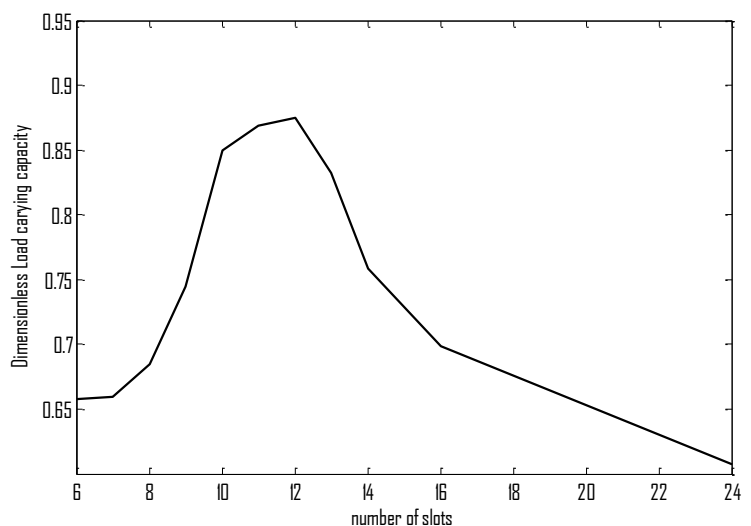

Fig. 12: number of slots vs load carrying capacity for double rows symmetry configuration

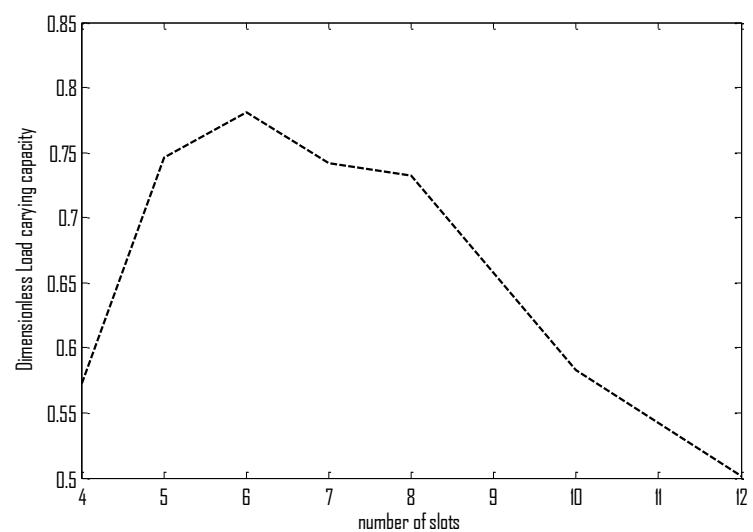

Fig. 13: number of slots vs load carrying capacity for single row asymmetry configuration

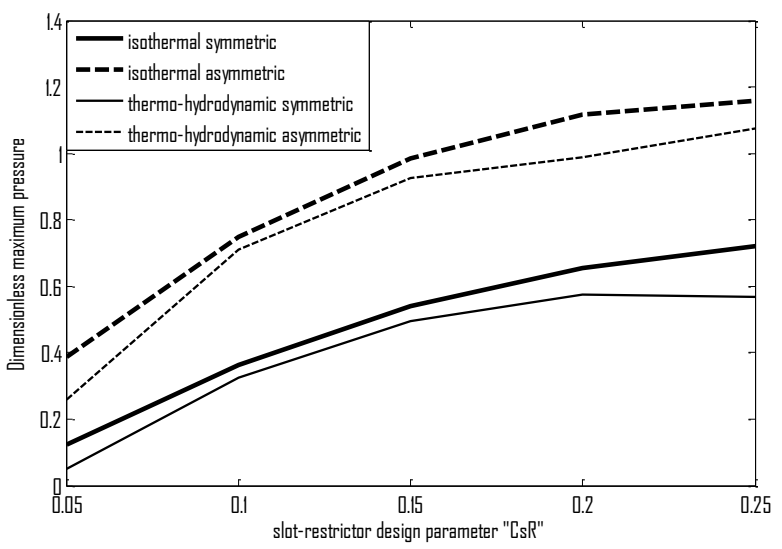

Fig. 14: Variation of dimensionless maximum pressure vs. slot-restrictor design parameter with different configurations for full thermohydrodynamic and isothermal solutions 


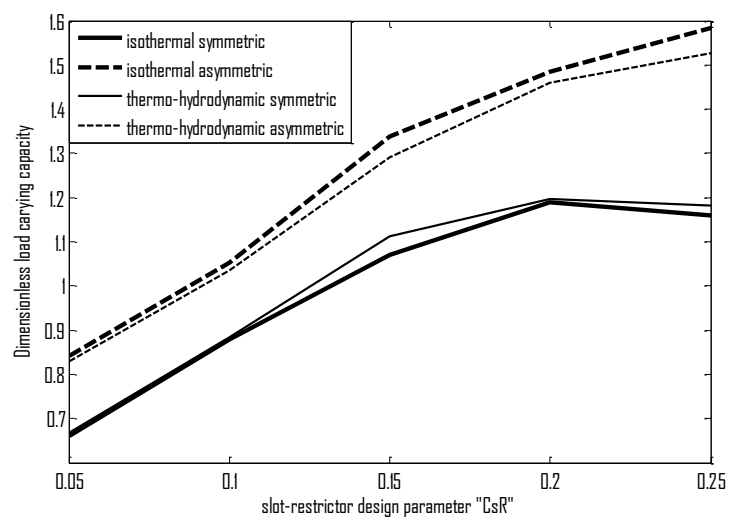

Fig. 15: Variation of dimensionless load carrying capacity vs. slotrestrictor design parameter with different configurations for full thermohydrodynamic and isothermal solutions

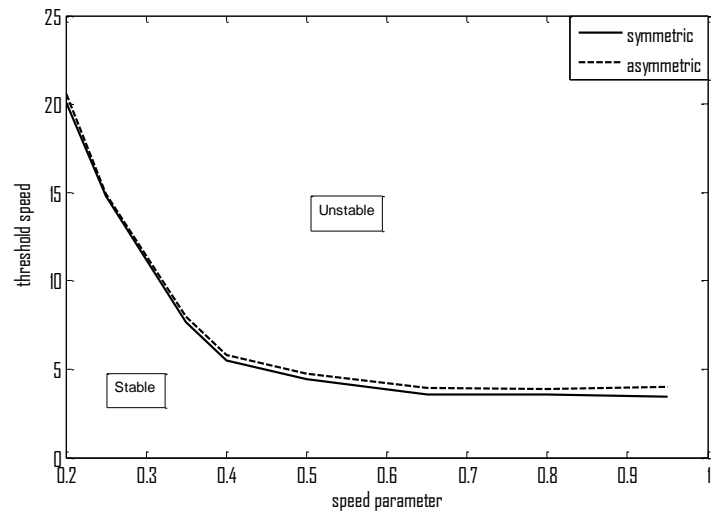

Fig.16: Variation of stability threshold speed margin

\section{Conclusion}

An analytical study on the performance of slot-entry hybrid journal bearings has been carried out. It was concluded that: 1-Considering the load carrying capacity, the slot-entry hybrid bearing with asymmetric configuration give a better performance than that of the symmetric configuration for the same eccentricity ratio.

2-The number of slots is an important factor that affects the bearing performance; in the case of symmetry configuration, the optimum load carrying capacity is attained using double row with 12 slots per row, while it is attained with single row 6 slots for asymmetry configuration.

3-The performance of the hybrid bearings depends on the use of optimum control parameters whereas the asymmetric configuration is generally advantageous.

4-The stability of hybrid bearings can also be modified by using the suitable geometry configuration. It is observed that the slot-entry hybrid journal bearing with asymmetric configuration is more stable than that with symmetric configuration.

\section{References}

[1] Dowson D, "A generalized Reynolds equation for film fluid lubrication". Int. Journal Mech. Sc. Pergamon Press. Vol. 4, pp. 159-170, 1962.
[2] Satish C. Sharma, Vijay Kumar, S.C. Jain, R. Sinhasan,

"A study of slot-entry hydrostatic/hybrid journal bearing using the finite element method". Tribology International 32 (1999) 185-196.

[3] Satish C. Sharma,S.C. Jain, N. Madhu Mohan Reddy," Influence of elastic effects on the performance of slot- entry journal Bearings". Tribology International 32 (1999) 537551

[4] H. Hirani, K. Athre, S. Biswas, "A Hybrid Solution Scheme for Performance Evaluation of Crankshaft Bearings", Journal of Tribology, Vol.122/733(2000).

[5] Satish C. Sharma, Vijay Kumar, S.C. Jain, T. Nagaraju, Giriraj Prasad," Thermo-hydrostatic analysis of slot- entry hybrid journal bearing". Tribology International 35 (2002) 561-577.

[6]Zhang Qide," Fluid bearing spindles for data storage devices", a thesis, National University of Singapore, 2003.

[7] Cheng-HsienChen ,Ching-Chu Huang, "The influences of orifice restriction and journal eccentricity on the stability of the rigid rotor-hybrid bearing system", Tribology International 37 (2004) 227-234.

[8]Julio Gomez-Mancilla, Valeri Nosov, Gerardo SilvaNavarro. "Rotor-Bearing System Stability Performance Comparing Hybrid versus Conventional Bearings". International Journal of Rotating Machinery 2005:1, 16-22.

[9]H. C. Garg, "Performance of Asymmetric Slot-Entry Hybrid Journal Bearing Operating with Non-

Newtonian Lubricant", Journal of Engineering and Technology, | Jan-Jun 2011 | Vol 1 | Issue 1.

[10]Vikas M.Phalle, Satish C.Sharma,S.C.Jain, "Performance analysis of a 2-lobe worn multirecess

hybrid journal bearing system using different flow control devices". Tribology International 52 (2012) 101-116.

[11] Prashant .Kushare, Satish C.Sharma. "A study of 2lobe symmetric hole entry hybrid journal bearing Operating with non-Newtonian lubricant considering thermal effects". Tribology International 92(2015)567-576.

[12] Rowe, "Hydrostatic and hybrid bearing design" Publishing Co., Butterworth, 1983.

About Author (s):

A. Abdelbadie : M.Sc. Mechanical Engineering, Engineering consultant at Alexandria Port Maritime, Egypt.

W.A. Crosby : Professor, Mechanical Engineering Department, Faculty of Engineering, Alexandria University, Egypt

I.M. ElFahham : Associate Professor, Mechanical Engineering Department, Faculty of Engineering, Alexandria University, Egypt 